\title{
A study of the first harmonic of the large-scale anisotropies with the KASCADE-Grande experiment
}
A. Chiavassa*3, W.D. Apel ${ }^{1}$, J.C. Arteaga-Velázquez ${ }^{2}$, K. Bekk ${ }^{1}$, M. Bertaina ${ }^{3}$, J. Blümer ${ }^{1,4}$, H. Bozdog ${ }^{1}$, I.M. Brancus ${ }^{5}$, E. Cantoni ${ }^{3,6}$, F. Cossavella $^{4}$, K. Daumiller ${ }^{1}$, V. de Souza ${ }^{7}$, F. Di Pierro ${ }^{3}$, P. Doll ${ }^{1}$, R. Engel ${ }^{1}$, D. Fuhrmann ${ }^{8}$, A. Gherghel-Lascu ${ }^{5}$, H.J. Gils ${ }^{1}$, R. Glasstetter ${ }^{8}$, C. Grupen ${ }^{9}$, A. Haungs ${ }^{1}$, D. Heck ${ }^{1}$, J.R. Hörandel ${ }^{10}$, D. Huber ${ }^{4}$, T. Huege ${ }^{1}$, K.-H. Kampert ${ }^{8}$, D. Kang ${ }^{4}$, H.O. Klages ${ }^{1}$, K. Link ${ }^{4}$, P. Łuczak ${ }^{11}$, H.J. Mathes ${ }^{1}$, H.J. Mayer ${ }^{1}$, J. Milke ${ }^{1}$, B. Mitrica ${ }^{5}$, C. Morello ${ }^{6}$, J. Oehlschläger ${ }^{1}$, S. Ostapchenko ${ }^{12}$, N. Palmieri ${ }^{4}$, T. Pierog ${ }^{1}$, H. Rebel ${ }^{1}$, M. Roth ${ }^{1}$, H. Schieler ${ }^{1}$, S. Schoo $^{1}$, F.G. Schröder ${ }^{1}$, O. Sima ${ }^{13}$, G. Toma ${ }^{5}$, G.C. Trinchero ${ }^{6}$, H. Ulrich ${ }^{1}$,

\section{A. Weindl ${ }^{1}$, J. Wochele ${ }^{1}$, J. Zabierowski ${ }^{11}$ - KASCADE-Grande Collaboration}

${ }^{1}$ Institut für Kernphysik, KIT - Karlsruhe Institute of Technology, Germany

${ }^{2}$ Universidad Michoacana, Inst. Física y Matemáticas, Morelia, Mexico

${ }^{3}$ Dipartimento di Fisica, Università degli Studi di Torino, Italy

${ }^{4}$ Institut für Experimentelle Kernphysik, KIT - Karlsruhe Institute of Technology, Germany

${ }^{5}$ Horia Hulubei National Institute of Physics and Nuclear Engineering, Bucharest, Romania

${ }^{6}$ Osservatorio Astrofisico di Torino, INAF Torino, Italy

${ }^{7}$ Universidade São Paulo, Instituto de Física de São Carlos, Brasil

${ }^{8}$ Fachbereich Physik, Universität Wuppertal, Germany

${ }^{9}$ Department of Physics, Siegen University, Germany

${ }^{10}$ Dept. of Astrophysics, Radboud University Nijmegen, The Netherlands

${ }^{11}$ National Centre for Nuclear Research, Department of Astrophysics, Lodz, Poland

${ }^{12}$ Frankfurt Institute for Advanced Studies (FIAS), Frankfurt am Main, Germany

13 Department of Physics, University of Bucharest, Bucharest, Romania

E-mail: andrea.chiavassaeto.infn.it

\begin{abstract}
We present the results of a search for large scale anisotropy in the arrival direction of cosmic rays performed with the KASCADE-Grande experiment at energies greater than $10^{15} \mathrm{eV}$. To eliminate spurious anisotropies due to atmospheric or instrumental fluctuations we apply the East-West method. The count distribution in 20 minutes bins of sidereal time are analyzed searching for a dipole component: the significance of the amplitude of the first harmonic is $3.5 \sigma$, therefore an upper limit is derived. The phase of the first harmonic, determined with an error of few hours, agrees with the measurements, obtained in the $10^{14}<E<2 \times 10^{15} \mathrm{eV}$ and $10^{17}<E<$ $10^{18} \mathrm{eV}$, energy ranges, by the EAS-TOP, IceCube, IceTop and Pierre Auger experiments. Thus the KASCADE-Grande experiment results are filling the gap between the previously cited results confirming the indication of a change of the first harmonic phase at energies greater than $\sim 2 \times$ $10^{14} \mathrm{eV}$.
\end{abstract}

The 34th International Cosmic Ray Conference,

30 July- 6 August, 2015

The Hague, The Netherlands

\footnotetext{
* Speaker.
} 


\section{Introduction}

Recent measurements of the cosmic-ray spectrum in the energy range around the "knee", i.e. the change of slope from $\gamma \sim-2.7$ to $\gamma \sim-3.1$, have shown that this feature can be interpreted in terms of lack of the primary particles containment inside magnetic fields. In such context the knee at $2-4 \times 10^{15} \mathrm{eV}$ is attributed to light elements ( $\mathrm{H}$ or $\mathrm{He}$ ) [1, 2], while the spectrum of heavier elements shows a change of slope at higher energies [3,4]. All these results are based on the measurement of the mass group spectra (from two to five) of primary cosmic rays. These studies cannot discriminate between two different astrophysical scenarios explaining the knee either as the limit of acceleration in galactic sources or as a propagation effect. A measurement that can separate these two scenarios is the search for large scale anisotropies in the arrival direction of primary cosmic rays.

In the energy range from $1-20 \mathrm{TeV}$ different experiments, such as Tibet-III [5], MILAGRO [6], ARGO-YBJ [7], IceCube [8] and IceTop [9], detected both large-scale and small-scale anisotropies. At higher energies statistically significant detection has been claimed by the EASTOP [10], IceCube [11] and IceTop [9] experiments. In this energy range the amplitude of the large scale anisotropy is at the level of $\sim 10^{-3}$.

The statistical significance of anisotropy search depends on the number of events in the data set, thus long duration data taking are needed. But the counting rates of EAS experiments are affected by instrumental and atmospheric effects (such as pressure or temperature variations) that are orders of magnitudes larger than those expected by cosmic-ray anisotropies. A method to take into account these variations is the East-West method [12]: an algorithm based on the counting rate differences between East-ward and West-ward arrival directions.

\section{The Experiment}

The multi-detector experiment KASCADE [13] (located at $49.1^{\circ} \mathrm{N}, 8.4^{\circ} \mathrm{E}, 110 \mathrm{~m}$ a.s.l.) was extended to KASCADE-Grande in 2003 by installing a large array of 37 stations (named Grande) consisting of $10 \mathrm{~m}^{2}$ plastic scintillation detectors each (Fig. 1). KASCADE-Grande provided a sensitive area of about $0.5 \mathrm{~km}^{2}$ and operated jointly with the existing KASCADE detectors. The active data taking ended in January 2013. The Grande array was installed on an irregular triangular grid with an average spacing of $137 \mathrm{~m}$. For triggering purposes the stations were organized in 16 hexagons with six stations at the edges and one in the center. The trigger was given by the full coincidence of the seven modules of a single hexagon. The analysis discussed in this article is based on the data recorded by the Grande array.

The event arrival direction is obtained fitting the particle arrival time measured by the 37 Grande stations. The core location, the slope of the lateral distribution function and the shower size (i.e. the total number of charged particles $N_{c h}$ ) are the result of a maximum likelihood fit, comparing the measured number of particles with the one expected from an NKG-like lateral distribution function [14] of the charged particles in the EAS.

KASCADE-Grande provides the unique opportunity of evaluating the reconstruction accuracies of the Grande array by a direct comparison with an independent experiment. For a subsample of events the two independent reconstructions of the KASCADE and the Grande arrays are com- 


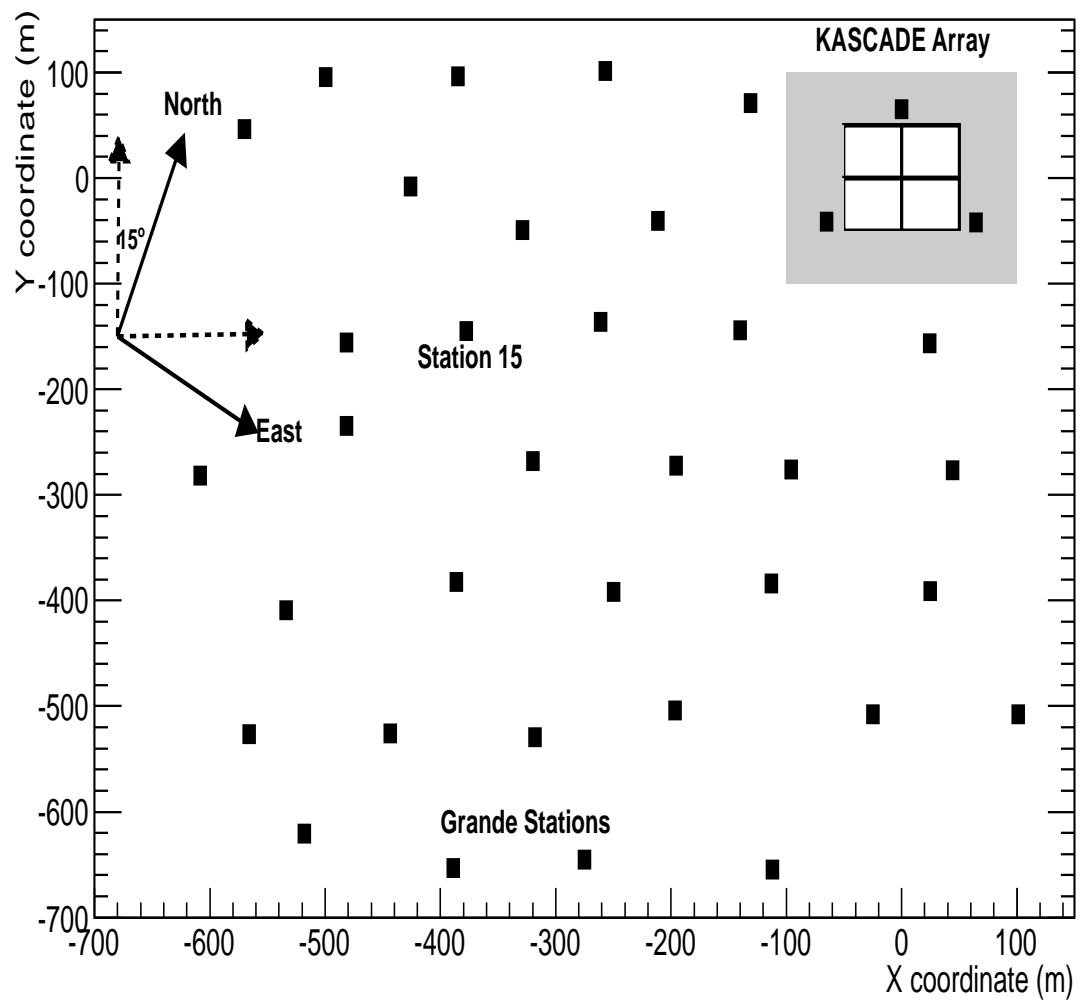

Figure 1: Layout of the KASCADE-Grande experiment. The position of Station 15 is shown (see text).

pared obtaining that the Grande reconstruction accuracies, above the 100\% reconstruction and detection efficiency, are: $\leq 15 \%$ for $N_{c h}$ (with a systematic shift respect to KASCADE $\leq 5 \%$ ); $\sim 0.8^{\circ}$ for the arrival direction; and $\sim 6 \mathrm{~m}$ for the core position.

A detailed description of the event reconstruction and performances of the Grande array can be found in [15].

\subsection{Data selection}

The number of counts from the East and West ward sectors are affected by the trigger inefficiency in the same way, therefore the East-West method can be applied also to data collected with trigger conditions not reaching a 100\% efficiency. Therefore no selection cuts are applied on the core position and all the events with zenith angle $\theta \leq 40^{\circ}$ and shower size $N_{c h} \geq 10^{5.2}$ are used in this analysis. The azimuthal angle distributions were used as check of the data quality, the only anomaly that was found is in the distribution of the events having the largest particle density measured by station number 15: therefore these events are rejected in the following analysis.

The primary energy is estimated from the shower size $N_{c h}$, first calculated at a reference zenith angle (applying constant intensity cut technique [16]) and then converted to energy using the relation obtained in [17], for primary protons, from a complete EAS simulation based on the QGSJetII- 
02 hadronic interaction model [18]. The values of the amplitude and the phase of the first harmonics will be referred to the median energy of the event sample. Having calibrated the relation from $N_{c h}$ to energy for primary protons the energy we estimate is a lower limit of the true one, as the chemical composition of real events is heavier than pure protons.

The distribution of the number of events in 20 minutes intervals of solar time is shown by the solid line in figure 2: large fluctuations, due to spurious anisotropies introduced by atmospheric and instrumental effects, are present. The dashed line shows the same distribution obtained applying the East-West method: the previously mentioned spurious fluctuations are removed.

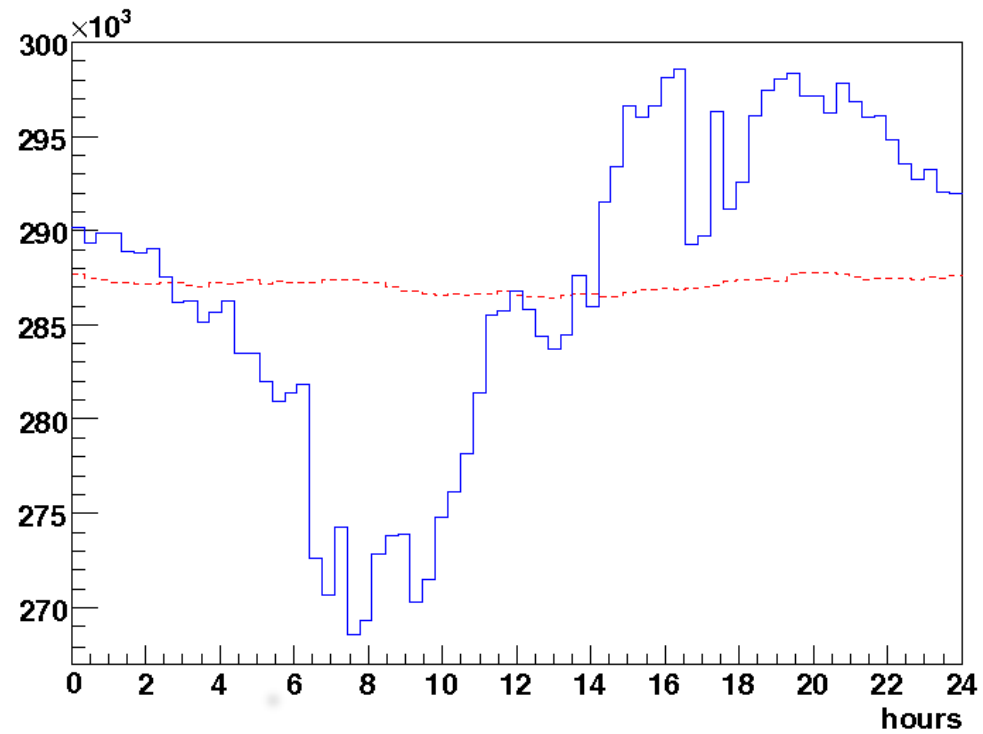

Figure 2: The solid line shows the solar time distribution of the number of events in 20 minutes intervals; the dashed line is the same distribution having applied the East-West method.

\section{Results and discussion}

The values of the amplitudes and phases of the first harmonic analysis of the counts distributions (with 20 minutes binning) in solar, sidereal ${ }^{1}$ and anti-sidereal times are reported in table 1. The Rayleigh probability that the sidereal time amplitude (shown in figure 3 ) is due to a fluctuation of the background is $0.2 \%$, its significance is $3.5 \sigma$, therefore we calculate, according to the distribution drawn from a population characterized by an anisotropy of unknown amplitude and phase as derived by Linsley [19], the $99 \%$ confidence level upper limit to the amplitude: $A \leq 0.47 \times 10^{-2}$.

These values are obtained using the full KASCADE-Grande statistics, therefore we cannot expect to obtain significant amplitudes dividing the event sample in energy intervals, we nevertheless perform this search to study the behavior of the phase with energy. The previously discussed analysis is therefore repeated in three intervals of $N_{c h}: 5.2 \leq \log N_{c h} \leq 5.6,5.6 \leq \log N_{c h} \leq 6.4$ and

\footnotetext{
${ }^{1}$ sidereal time: Common time scale among astronomers which is based on the Earth's rotation measured relative to the fixed stars.
} 


\begin{tabular}{|l|c|c|c|}
\hline time & $A \times 10^{-2}$ & hours & $\mathrm{P}$ \\
\hline sidereal & $0.28 \pm 0.08$ & $15.1 \pm 1.1$ & $0.2 \%$ \\
\hline solar & $0.15 \pm 0.08$ & $23.9 \pm 2.1$ & $17 \%$ \\
\hline anti-sidereal & $0.02 \pm 0.08$ & $1.8 \pm 14.4$ & $96 \%$ \\
\hline
\end{tabular}

Table 1: Results of first harmonic analysis (amplitude, phase and Rayleigh probability) in sidereal, solar and anti-sidereal time.

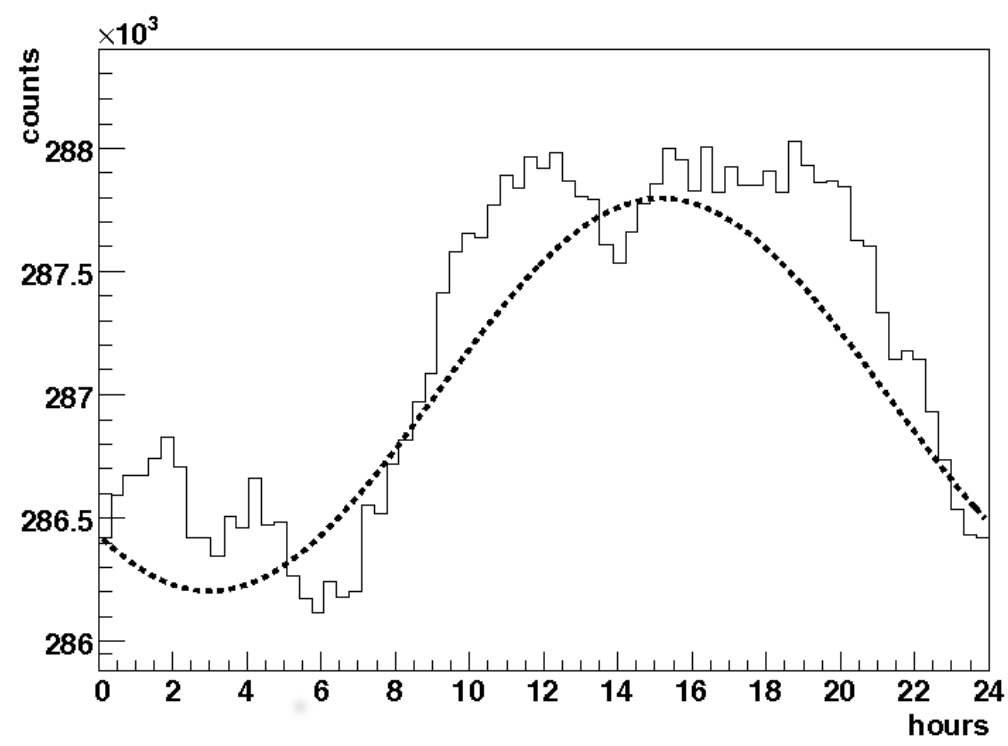

Figure 3: Distribution of the number of counts, obtained applying the East-West method, in 20 minutes intervals of sidereal time. The dashed line represent the calculated first harmonic: the amplitude and phase values are reported in table 1

$\log N_{c h} \geq 6.4$ : the obtained values are reported in table 2 together with the Rayleigh probabilities and the median energies.

\begin{tabular}{|l|c|c|c|}
\hline $\log \left(N_{c h}\right.$ & Median Energy $(\mathrm{eV})$ & $A \times 10^{-2}$ & Phase \\
\hline $5.2-5.6$ & $2.7 \times 10^{15}$ & $0.26 \pm 0.10$ & $225 \pm 22 \%$ \\
\hline $5.6-6.4$ & $6.1 \times 10^{15}$ & $0.29 \pm 0.16$ & $227 \pm 30 \%$ \\
\hline$\geq 6.4$ & $3.3 \times 10^{16}$ & $1.2 \pm 0.9$ & $254 \pm 42 \%$ \\
\hline
\end{tabular}

Table 2: Results of harmonic analysis in sidereal time performed in different intervals of $N_{c h}$

In the two panels of figure 4 the sidereal time distributions of the number of counts are shown. The significance of the first harmonic amplitude decreases with increasing the number of charged particles, therefore the corresponding phase is defined with a larger incertitude as confirmed by the error values.

Figures 5 and 6 show a comparison of the results presented in this work with those obtained at lower and upper energies. The phases of the first harmonic measured by KASCADE-Grande 

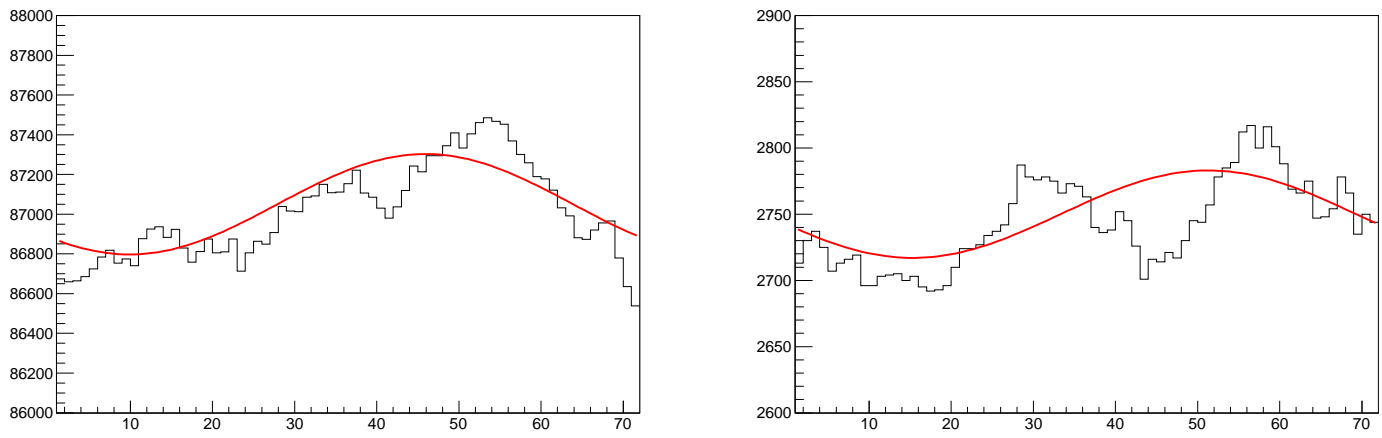

Figure 4: Sidereal time distribution of the number of counts, obtained, in the $10^{5.6}<N_{c h}<10^{6.4}$ (left panel) and $N_{c h}>6.4$ (right panel) shower size intervals, applying the East-West method ( $\Delta t=20$ minutes). The line represent the calculated first harmonic: amplitudes and phases are reported in table 2

point the same sky region indicated by measurements obtained at energies greater than $10^{14} \mathrm{eV}$ by the EAS-TOP, IceCube and IceTop experiments and at energies between $2 \times 10^{16}$ and $10^{18} \mathrm{eV}$ by the Pierre Auger Observatory [20,21]. These results are thus filling the energy gap between the knee and the ankle of the cosmic rays spectrum, confirming (still with large error bars) that in this energy range the phase of the first harmonic is different from the one measured below $10^{14} \mathrm{eV}$ and above $10^{18} \mathrm{eV}$.

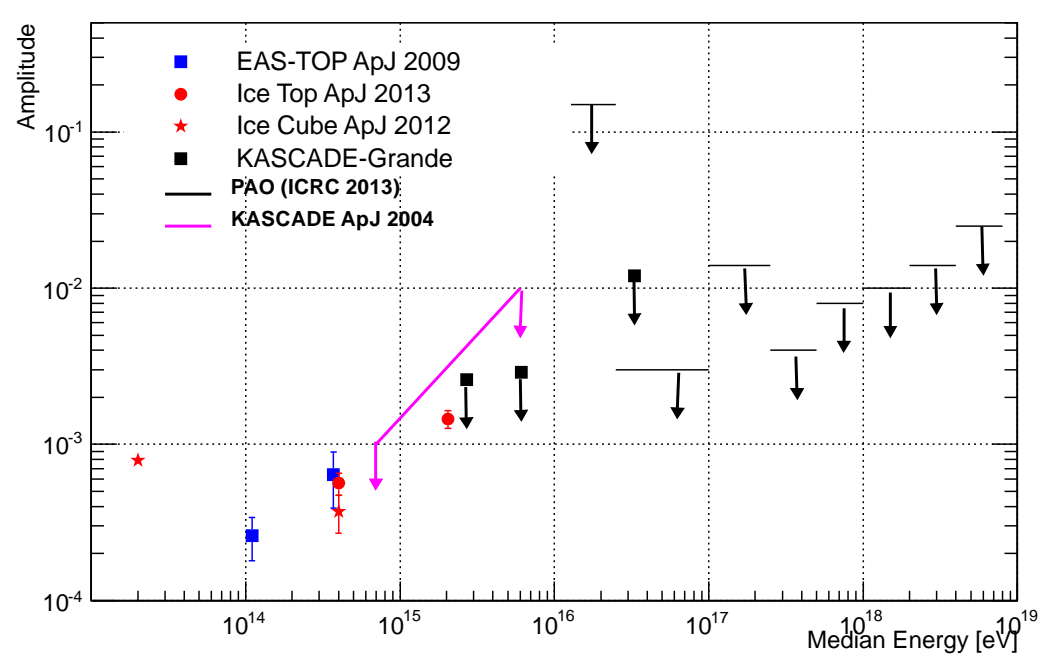

Figure 5: Comparison of the upper limits to the amplitude of the first harmonic obtained by KASCADEGrande with experimental results in the $10^{13}<E<10^{19} \mathrm{eV}$ energy range.

\section{References}

[1] T. Antoni et al., Astropart. Phys. 16, 373 (2002). 


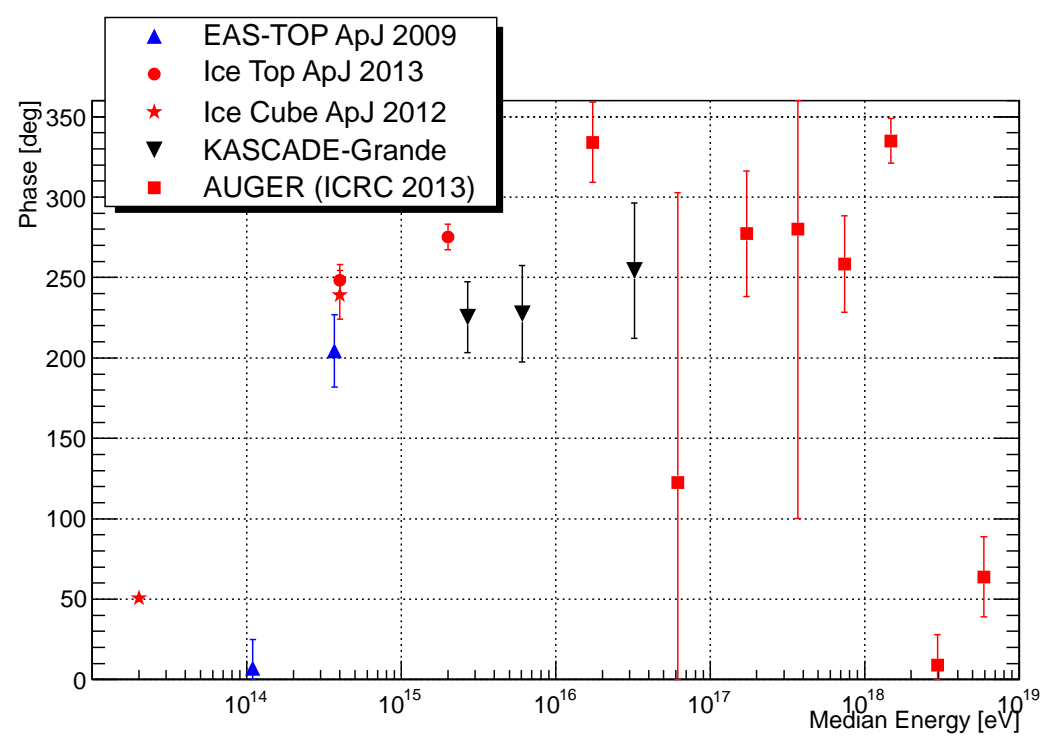

Figure 6: Comparison of the KASCADE-Grande measurements of the phase of the first harmonic with experimental results obtained in the $10^{13}<E<10^{19} \mathrm{eV}$ energy range.

[2] M. Aglietta et al., Astropart. Phys. 21, 223 (2004).

[3] T. Antoni et al., Astropart. Phys. 24, 1 (2005).

[4] W.D. Apel et al., Phys. Rev. Lett. 107, 171104 (2011).

[5] M. Amenomori et al., ApJ Lett. 626, L29-L32 (2005).

[6] A. A. Abdo et al., ApJ 698, 2121-2130 (2009).

[7] B. Bartoli et al., Phys. Rev. D 88. 082001 (2013).

[8] R. Abbasi et al., ApJ Lett. 718, L194-L198 (2010).

[9] M. G. Aartsen et al., ApJ 765, 55 (2013).

[10] M. Aglietta et al., ApJ 692, L130-L133 (2009).

[11] R. Abbasi et al., ApJ 746, 33 (2012).

[12] R. Bonino et al., Astrophys. J. 738, 67 (2011).

[13] T. Antoni et al., Nucl. Instr. \& Meth. A 513, 429 (2003).

[14] W.-D. Apel et al., Astropart. Phys. 24, 467 (2006).

[15] W.D. Apel et al., NIM A 620 (2010) 202.

[16] J. Hersil et al., Phys. Rev. Lett. 6, 22 (1961).

[17] D. Kang et al., Proc. of the $31^{\text {st }}$ ICRC Lodz (Poland), \#icrc1044 (2009).

[18] S. Ostapchenko, Phys. Rev. D 74, 014026 (2006).

[19] J. Linsley, Phys. Rev. Lett. 34, (1975) 1530.

[20] I. Sidelnik et al., Proc. of the $33^{\text {rd }}$ ICRC Rio de Janeiro (Brasil), (2013).

[21] A. Aab et al., arXiv:1411.6953v1 (2014). 